\title{
The Impact of Cloud Computing-Based Big Data Platform on IE Education
}

\author{
Ziyan Wang, ${ }^{1}$ Yijie Wan $\mathbb{D}^{2}{ }^{2}$ and Hua Liang ${ }^{3}$ \\ ${ }^{1}$ Office of Academic Affairs, Hunan International Economics University, Changsha 410205, Hunan, China \\ ${ }^{2}$ College of Media and Communications, Guangxi University for Nationalities, Nanning 530000, Guangxi, China \\ ${ }^{3}$ School of Information and Mechatronics Engineering, Hunan International Economics University, Changsha 410205, \\ Hunan, China \\ Correspondence should be addressed to Yijie Wan; 20100039@gxun.edu.cn
}

Received 24 November 2021; Revised 23 December 2021; Accepted 6 January 2022; Published 27 January 2022

Academic Editor: Haibin Lv

Copyright (c) 2022 Ziyan Wang et al. This is an open access article distributed under the Creative Commons Attribution License, which permits unrestricted use, distribution, and reproduction in any medium, provided the original work is properly cited.

\begin{abstract}
"Mass Entrepreneurship, Mass Innovation" is for the benign, rapid, and better development of our economy and society. In the 21 st century, innovation and entrepreneurship (IE) under the background of technological development must take the masses as the main body to stimulate the vitality of mass IE. Compared with traditional IE education, the impact of big data platforms on education cannot be underestimated. Therefore, this article aims to research the influence of cloud computing-based big data platforms on IE education. This article proposes to use the cloud computing-based big data platform as a carrier to carry out IE education to change the drawbacks of traditional IE education. This research uses the literature analysis method and questionnaire survey method. It takes college students as the survey subjects of IE education, who are also the main object, and fully collects data. The research shows that cloud computing-based big data platforms have multiple impacts on IE education. During the epidemic, noncontact education is closely related to network technology.
\end{abstract}

\section{Introduction}

In the 21st century, open to the outside world for more than 40 years, domestic colleges and universities have started to carry out IE education for more than a decade. Many colleges and universities have tried different directions in exploring IE education, and they have achieved some results. However, there is still a clear gap between the current situation of IE education and my country's national development strategy goals and actual needs. Therefore, continuing to carry out research on IE education related to the development of the times has very theoretical and practical value.

To enrich the theoretical system of IE education in colleges, this article focuses on the collection and sorting of related documents in the past ten years. Based on this premise, it makes an in-depth analysis of the connotation and extension of the relevant theoretical concepts of IE education. The current trouble in the reform of IE education in colleges is discussed, regional characteristics are combined to focus on exploring the difficulties in the change of IE education in Tianjin colleges, and solutions are proposed. The theoretical research on university IE education for follow-up scholars has the value for reference.

Under the tide of reforms in the new era, IE education is more and more valued by the Chinese people, but there are not too many relevant documents at home and abroad. Peris-Ortiz et al. verified the influence of the professor's entrepreneurial spirit on the entrepreneurial activities of 418 college students. The experimental method uses different algorithms to analyze the differences among the three countries. However, the selection and screening of sample size has unmanageable randomness [1]. Wei et al. encourages service providers to choose the best bidding strategy based on overall utility to maximize profits. Based on the unit prices of different types of resources, a resource allocation model is proposed to ensure the best interests of infrastructure suppliers [2]. Considering the emergence of 
cloud computing, in order to sort the search results and protect the privacy of the correlation score between keywords and files, Zhang et al. proposed a novel addition sequence and privacy protection function family [3]. Jin L et al. solve this challenging problem by proposing a new attribute-based data-sharing solution, which is suitable for mobile users with limited resources in cloud computing. Experimental results believe that the proposed scheme is proven to safely resist adaptive selection ciphertext attacks, which is widely regarded as a standard security concept [4]. As a big data platform that supports analysis, there are not a few related studies so far. Li and Feng believed that, in the current era, the use of replication technology in parallel and distributed computer systems can improve system performance and availability. They proposed an efficient big data platform that can effectively support massive data integration and data mining algorithms and tested the effectiveness of the algorithms in artificial simulation data sets and UCI machine learning databases. From the perspective of visualization and numerical simulation, the results prove that the algorithm is effective [5]. Martin's main goal in the experiment was to design a platform to accomplish as many goals as possible, such as collection, sorting, and transmission [6]. Chen and others explored smart city community governance research based on the blockchain big data platform. This can be more intuitively expressed [7]. Li et al. focused on visualization. As an important part of visualization, they proposed 3D mesh reconstruction (R3MR) based on regional growth in the big data platform [8]. Generally speaking, due to the wide application and many technical points, there are many and very exciting related research projects on big data platforms, and there are also many research projects on cloud computing, but there is still relatively little research on the topic of this article.

Relevant research on the impact of IE education has been discussed at home and abroad, but this article uses cloud computing-based big data platform as the main point of discussion, which is not available in related research institutes. In this article, the following innovations are mainly included. (1) Innovation in the research direction: this article focuses on analyzing cloud computing algorithms, focusing on the implementation process and big data platform, introducing its composition principle, and looking for reasons from within. (2) Innovation of the research object: because the main object of IE education is college students, the questionnaire survey method is used, and the questionnaires collected are all from college students, which can maintain the quality and guarantee the quantity.

\section{Method Introduction}

\subsection{Cloud Computing Clustering Algorithm}

2.1.1. Introduction to the K-Means Algorithm Based on Cloud Computing. The $\mathrm{K}$-means algorithm is very popular among scholars $[9,10]$. Because of its simplicity and efficiency, it is widely used, and therefore it was selected as one of the top 10 data mining algorithms. The execution process of the $\mathrm{K}$-means algorithm is as follows:
(1) Specify $k$ in advance (the final number of clusters)

(2) Calculate the distance from each object in the data set to each initial gathering point, select the shortest distance among them, and classify the object to the initial gathering point

(3) According to the result obtained using step 2, calculate the average value of each cluster and assign it to the initial cluster point

Repeat steps 2 and 3 until equation (1) converges.

$$
J={ }_{n}^{1} \sum_{i=1}^{k} \sum_{x \in C_{i}}\left|x-m_{i}\right|^{2}
$$

In formula (1), the data set $D$ contains $n$ data $x_{1}, x_{2}, x_{3}, \ldots, x_{n}$, which belong to the $\mathrm{d}$-dimensional space $R^{d} ; m_{i}$ indicates the initial gathering point of the cluster $C i$, and $k$ represents the number of prespecified clusters. The termination condition of the K-means algorithm is as follows: after repeating steps (2) and (3), the value of the initial gathering point remains unchanged [11].

2.1.2. Evaluation of Clustering Results. (1) Davies-Bouldin Coefficient. The coefficient can be calculated using equation (2):

$$
\mathrm{DB}={ }_{n}^{1} \sum_{i=1}^{n} \max _{j \neq i}\left(\frac{\sigma_{i}+\sigma_{j}}{d\left(c_{i}, c_{j}\right)}\right) .
$$

Among them, $n$ is the number of clusters, $c_{x}$ is the center of cluster $x, \sigma_{\mathrm{x}}$ is the average distance from all data points in $x$ to $c_{x}$, and $d\left(c_{i}, c_{j}\right)$ is the distance from the center of cluster $c_{i}$ to $c_{j}$ [12]. If an algorithm can obtain results with high intracluster similarity and low intercluster similarity, then the algorithm can obtain a lower Davies-Bouldin coefficient [13]. Based on this method, generally speaking, the lower the value obtained, the more satisfactory the results [14].

(2) Dunn Coefficient. The Dunn coefficient calculates the similarity between the cluster and the benchmark classification [15]. The Dunn coefficient can be calculated using equation (3):

$$
D=\frac{\min _{1 \leq i \leq j \leq n} d(i, j)}{\max _{1 \leq k \leq n} d^{\prime}(k)} .
$$

Among them, $d(i, j)$ represents the distance between clusters $i$ and $j, d^{\prime}(k)$ represents the internal distance of cluster $k$, and the distance between clusters $i$ and $j$ can be any two points in the two clusters. The distance between two clusters is the distance between the gathering points of the two clusters. Similarly, $d^{\prime}(k)$ can also be measured in a variety of ways, such as the maximum distance between any two points in the cluster $k$. Generally speaking, an algorithm that can obtain a high Dunn coefficient can be considered a good algorithm $[16,17]$.

(3) Rand Method. The Rand coefficient calculates the resemblance between the cluster and the benchmark 
classification. The Rand coefficient can also be used to measure the percentage of correct decisions made by the algorithm [18]. The Rand coefficient can be calculated using equation (4):

$$
\mathrm{RI}=\frac{\mathrm{TP}+\mathrm{TN}}{\mathrm{TP}+\mathrm{FP}+\mathrm{FN}+\mathrm{TN}}
$$

In the formula, the number of true positives is represented by TP, TN represents the number of true negatives, FN corresponds to TP, and FN corresponds to TN. In some clustering applications, this may result in undesirable features. F-measure solves this problem.

(4) F-Measure. First, we can calculate the accuracy, which is defined as the ratio of the number of samples correctly classified by the classifier to the total number of samples for a given test data set. That is, the accuracy of the test data set when the loss function is $0-1$ loss. It uses the parameter $\beta \geq 0$ to weight the recall rate. The precision and recall are defined using formulas (5) and (6):

$$
\begin{aligned}
& P=\frac{\mathrm{TP}}{\mathrm{TP}+\mathrm{FP}}, \\
& R=\frac{\mathrm{TP}}{\mathrm{TP}+\mathrm{FN}} .
\end{aligned}
$$

Among them, $P$ represents the precision rate, and $R$ represents the recall rate. We can use equation (7) to calculate $F$-measure:

$$
F_{\beta}=\frac{\left(\beta^{2}+1\right) \cdot P \cdot R}{\beta^{2} \cdot P+R} .
$$

Pay special attention to the situation of $\beta=0$ and $F_{0}=P$. However, when $\beta=0, R$ has no effect on $F$-measure. As $\beta$ increases, the weight of recall rate in $F$-measure will also increase. The calculation of the corresponding $F$-measure will be easier.

(1) Jaccard Coefficient. The Jaccard coefficient is used to quantify the resemblance between $\mathrm{A}$ and $\mathrm{B}$ data sets. The value range of Jaccard coefficient is $0-1$. When the value is 1 , it means that the two data sets are exactly the same. When the value is 0 , it means that any data in the two data sets are different [19]. The Jaccard coefficient is defined using equation (8):

$$
J(A, B)=\frac{|A \cap B|}{|A \cup B|}=\frac{\mathrm{TP}}{\mathrm{TP}+\mathrm{FP}+\mathrm{FN}} .
$$

(2) Fowlkes-Mallows Coefficient. The Fowlkes-Mallows coefficient is used to calculate the resemblance between the clustering algorithm returned and the benchmark classification. A high coefficient value represents a higher degree of similarity. The coefficient value can be calculated using equation (9):

$$
\mathrm{FM}=\frac{\mathrm{TP}}{\mathrm{TP}+\mathrm{FP}} \cdot \frac{\mathrm{TP}}{\mathrm{TP}+\mathrm{FN}} .
$$

TP represents the value of true positives, FP represents the value of false positives, $\mathrm{FN}$ represents the value of false negatives, and the FM coefficient is the geometric mean of the precision and recall rates $\mathrm{P}$ and R. F-measure is the harmonic mean [20]. In addition, the precision rate and recall rate are also called Wallace indicators $B^{\mathrm{I}}$ and $B \amalg$.

2.2. Big Data and LSTM Algorithm. In 1997, Hochreiter et al. first proposed a long short-term memory network model [21]. This network is a special RNN model, by linking each node to each other, as shown in Figure 1, which is the basic structure of RNN. In order to illustrate its structural characteristics, it is analyzed in the form of a graph. As shown on the right side of Figure 1, mis the input layer, $s$ is the hidden layer, $o$ is the output layer, $X$ is hidden layer, $Y$ is the input layer, $Z$ refers to the weight of the output layer, where the state of the $a$-th hidden layer is as follows:

$$
s_{a}=f\left(M * m_{a}+Z * s_{a-1}\right) .
$$

$s_{a-1}$ refers to the state of the previous hidden layer and $s_{a}$ is the state of the current hidden layer, so that the current input value can be connected with the previous calculation. If the network wants to form long-term memory, it needs to be combined with previous calculations and link the following equation:

$$
m_{a}=f\left(X * m_{a}+Z * s_{a-1}+Z_{2} * s_{a-2}+\cdots+Z_{n} * s_{a-n}\right) .
$$

However, this will cause the amount of calculation to increase exponentially, which will increase the calculation time of the model, so the RNN model group will not be directly used for long-term memory calculations. The long short-term memory network model is based on the original RNN network. Each level adds a valve as a node. This makes the model solve the problem of RNN computing long-term memory and time-consuming data, so it is quickly applied in various aspects. The basic structure of the long short-term memory network model is shown in Figure 2 [22].

The three gates of the long short-term memory network model are added to the original RNN model. They are output gate, input gate, and forget gate. The gates can be opened or closed with respect to the output result of the network state in the layer before the model reaches the threshold. As shown in Figure 2, the sigmoid function is used on the valve node, and the state of the door corresponding to the sigmoid layer is determined by the value; output 0 is closed, and output 1 is open. As the output result is already higher than the threshold, the output of this gate will be multiplied by the output of the current layer and input it to the next layer. On the contrary, it is forgotten, so it can affect the previous one by controlling the valve switch. The sequence of the first part of the intermediate module in Figure 2 (marked " 1 " in the figure) determines which part of the information will be discarded in the cell state. The value output by the function $\sigma$ is determined according to the state of each cell. " 1 " means all reserved, " 0 " means all discarded, and formula (12) means 4 cells states:

$$
f_{t}=\sigma\left(W_{f} \cdot\left[h_{t-1}, x_{t}\right]+b_{f}\right) \text {. }
$$

The second part of the middle module (labeled " 2 ") in Figure 2 is the updated cell state, which determines that the 

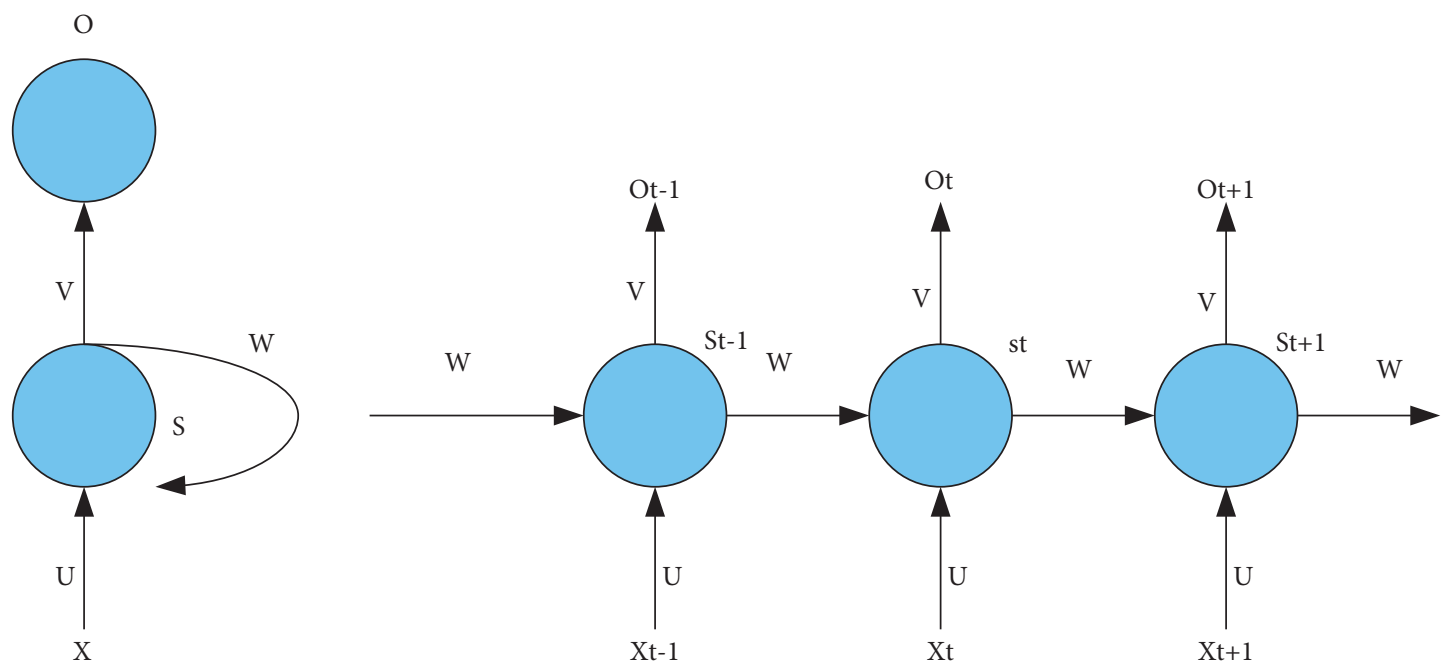

Figure 1: Basic structure of RNN.

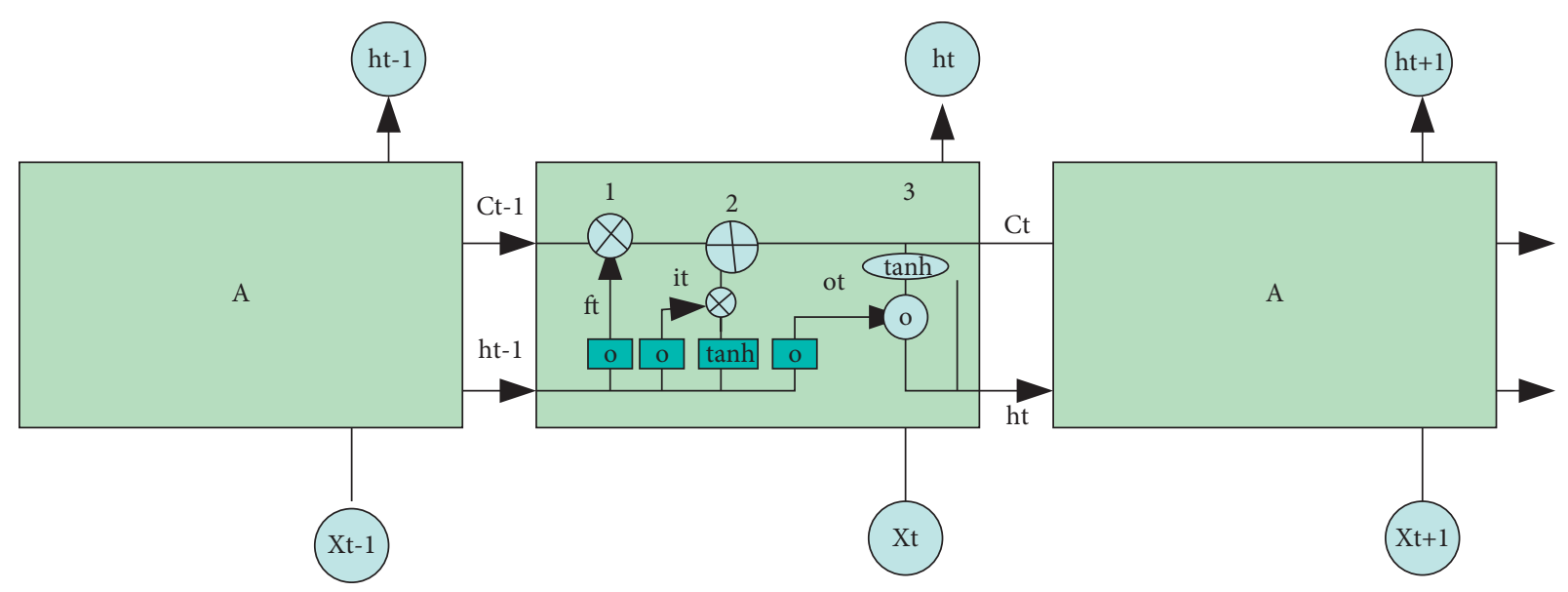

FIgURE 2: LSTM network structure.

different values of the three added levels need to be updated. $i_{t}$ and $\widetilde{C}_{t}$ can be calculated from these two layers.

$$
\begin{aligned}
i_{t} & =\sigma\left(W_{i} \cdot\left[h_{t-1}, x_{t}\right]+b\right), \\
\widetilde{C}_{t} & =\tanh \left(W_{C} \cdot\left[h_{t-1}, x_{t}+b_{C}\right]\right) .
\end{aligned}
$$

The third part of the middle module (marked as " 3 ") aims to update the cell network. The specific formula is as follows:

$$
C_{t}=f_{t} * C_{t-1}+i_{t} * \widetilde{C}_{t}
$$

At the end, the output value needs to be determined. The first step is to run the sigmoid function to determine which part to output. The second part processes the cell state through the tanh function and combines it with sigmoid. The output value $o_{t}$ is multiplied, and the output part $h_{t}$ we need is output:

$$
\begin{aligned}
& o_{t}=\sigma\left(W_{o} \cdot\left[h_{t-1}, x_{t}\right]+b_{o}\right), \\
& h_{t}=o_{t} * \tanh \left(C_{t}\right)
\end{aligned}
$$

The long short-term memory network model includes the process from input to output with the four main steps: (1) which part of the information needs to be discarded; (2) determining the information to be updated; (3) re-determining the cell state; and (4) input the value of the hidden layer, which is composed of the output value of the previous hidden layer and the value of the model input. The forgetting gate determines which part of the information needs to be input to the hidden layer, and the output gate determines which part of the information needs to be output. In this way, the sequence state is realized. Since invention, many scholars have made some subtle changes to the long shortterm memory network model, such as a variant proposed by Schmidhuber and Gers, which is a fluid long short-term memory network model variant with the addition of "peek." The "hole connection" technology is to receive the input value generated by the cell state in the sigmoid layer, and its basic structure is shown in Figure 3.

Input the cell state $C_{t-1}$ into each gate layer, when discarding and updating the information. It is necessary to consider the time at $t-1, t$, and $t+1$, namely, $h_{t-1}, x_{t}$, and 


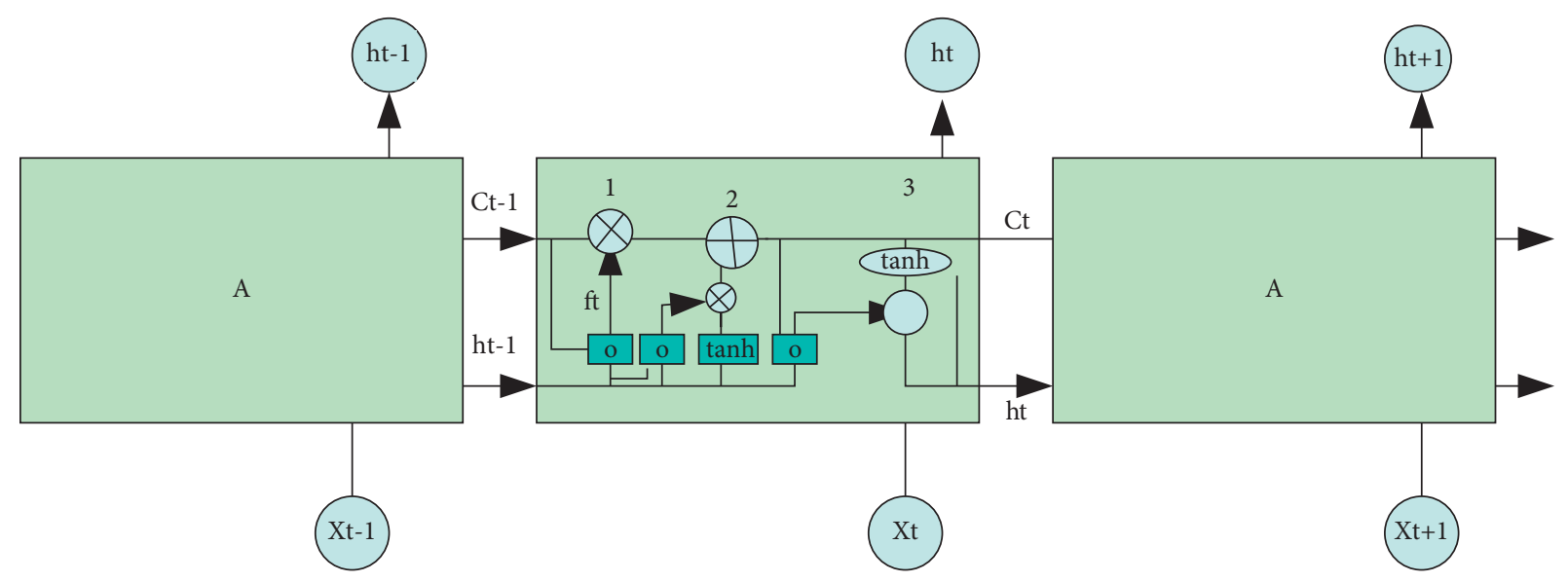

FIgURE 3: LSTM variant.

$C_{t-1}$ should be considered when discarding and updating the information, such as formulas (16)-(18):

$$
\begin{aligned}
f_{t} & =\sigma\left(W_{f} \cdot\left[C_{t-1}, h_{t-1}, x_{t}\right]+b_{f}\right), \\
i_{t} & =\sigma\left(W_{i} \cdot\left[C_{t-1}, h_{t-1}, x_{t}\right]+b_{i}\right), \\
o_{t} & =\sigma\left(W_{o} \cdot\left[C_{t-1}, h_{t-1}, x_{t}\right]+b_{0}\right) .
\end{aligned}
$$

The SBULSTM module considers the forward and backward states of the passenger flow time series at the same time and learns complex time features through superimposed recursive layers. Fully consider the quantitative relationship of big data. This completes the introduction of the algorithm, and the two algorithms will be combined and used in the experimental link.

\subsection{IE Education. IE education is a broad concept that can} be divided into two parts.

Innovative education refers to the use of educational theory to analyze innovative education, which means that educators exert influence on the educated through certain educational methods, so that they can form a unique ability to analyze and understand things and then become capable of new thinking and stubbornness. Understanding the independence of new things and laying a solid foundation for becoming innovative talents that the people and society need are relatively lacking in the society. In short, innovative education is not only a new concept of education in the new era but also a manifestation of educational ability. It aims to cultivate people's innovative ability through the implementation of different educational activities and cultivate what the party and the people need.

As for entrepreneurship education, the UNESCO has elaborated on the concept of entrepreneurship education: in a narrow sense, entrepreneurship education refers to the provision of resources and skills to help specific groups of people so that they can survive independently, especially for the poor; in a broad sense, it refers to the cultivation of pioneering talents for the future society, emphasizing the cultivation of management, skills, adventure, initiative, socialization, and independence. This kind of education is innovative, flexible, and continuous. What they have in common is that they all aim at cultivating and tapping people's entrepreneurial ability, potential, quality, and spirit as the ultimate goal.

From the development process of the two, in China, the foundation of entrepreneurship is innovation. In the 21st century, talents with innovative consciousness are needed. In the process of educating and becoming a talent, universities should follow the changing needs of the market and society and pay attention to the differences in individual development. The essence of innovative education is to focus on innovation based on the overall quality of human development. The cultivation of spirit is the education of the consciousness level and the foundation of entrepreneurship education. Entrepreneurship education focuses on the cultivation of people's entrepreneurial ability and pays attention to the process of people's realization of social value, which is the education of the behavioral level. Only when the subject with innovation consciousness has a certain ability to innovate and entrepreneurship, can it truly put innovation into practice and become an entity that promotes the development of productivity.

\section{Experiment and Analysis}

\subsection{Research Methods}

3.1.1. Literature Analysis Method. This research will collect domestic and foreign monographs and essays on opportunistic entrepreneurship and college student entrepreneurship education through library and online database access. The collected data will be sorted and analyzed to clarify the connotation, elements, and value of opportunistic entrepreneurship. To understand the current status of research and development on entrepreneurship education and entrepreneurial intentions of college students at home and abroad, this research will focus on the collected data and carefully explore the impact of cloud computing-based big data platforms on IE education in recent years to ensure it is logical and persuasive, and it will also earnestly learn from the experience of the predecessors and improve one's own 
shortcomings. Since the literature analysis method and the content analysis method have common objects and do not have direct contact with the people and events recorded in the literature, they are both called noncontact research methods. The law of content analysis realizes the scientific understanding of facts through quantitative analysis of literature and statistical description. The difference between the two is that the focus of analysis and the means of analysis are different. This article will roughly find out the influence of cloud computing platform on innovation and entrepreneurship education through literature analysis.

3.1.2. Questionnaire Survey Method. All data in this study come from the questionnaire "Impact of Cloud ComputingBased Big Data Platform on IE education" collected by Questionnaire Star. In the end, the 800 collected student questionnaires were screened through statistical software, the duplicate and invalid questionnaires were eliminated, and 681 valid surveys were screened out as the database of this research. Through questionnaire surveys and interviews, college students' opportunistic entrepreneurial intentions were measured, and sample data needed for the empirical research in this article were obtained.

\subsection{Data Analysis}

3.2.1. Basic Data. It can be seen from Table 1 that there are slightly more girls than boys. There are 354 girls, accounting for $59 \%$, and 246 boys, accounting for $41 \%$. In terms of source of birth, there are slightly more students in cities than in rural areas, with 206 from rural areas. Accounted for $34.4 \%, 392$ were from cities, accounting for $65.6 \%$; in terms of whether they were only children, 379 were only children, and 219 were not only children; 203 were freshmen, accounting for $33.8 \%$; 375 were sophomores, accounting for $62.5 \%$; 10 juniors, accounting for $1.7 \%$; and seniors 11 , accounting for $2.0 \%$; only 96 students have received entrepreneurship education, accounting for $16.1 \%$, and 502 students have not received entrepreneurship education, accounting for $83.9 \%$; this reflects that our school's entrepreneurial education has become popular, and this type of education has not yet attracted widespread attention among students. There are 182 students who have taken part-time jobs during their time at school, accounting for $30.4 \%$, and 416 students have not done part-time jobs, accounting for $69.6 \%$. There are 224 students whose parents or relatives had entrepreneurial experience, accounting for $37.2 \%$, whereas there are 376 students whose parents or relatives had no entrepreneurial experience, accounting for $62.7 \%$.

It is clear from Figure 4 that most of the survey samples are urban household registrations. Nowadays, most of the students are urban children, and there are also some parents who have entrepreneurial experience. It can be preliminarily considered that family education has a great influence on innovation and entrepreneurship education.

Figure 5 clearly reflects the composition of the questionnaire, all of which are college students, and there are 199 freshmen and 236 sophomores, accounting for the bulk of the total number.

Figure 6 reflects the current college students' understanding of IE education. It is obvious that more families are now having only one child, a minority of those who have taken part-time jobs during university, and fewer IE education have received education. This can also be highlighted. Most college students do not pay enough attention to IE education.

From Figure 7, we can get a general understanding of the situation of students' families. There are few laid-off and unemployed families. Most families are still based on work units, and some are self-employed enterprises. This also provides a good foundation for IE in the future. Family is also an indispensable part of IE education.

3.2.2. One-Way Analysis of Variance. In order to test the impact of college students' gender, birthplace, parent's occupation, and parents' and relatives' entrepreneurial experience on entrepreneurial intentions, this study uses one-way analysis of variance to analyze the control variables of the data. The basic idea of hypothesis testing is the principle of "small probability events," and its statistical inference method is a contradiction method with certain probability properties. The idea of small probability means that a small probability event basically does not happen in an experiment. The idea of contradiction is to first propose a test hypothesis and then use appropriate statistical methods to use the principle of small probability to determine whether the hypothesis is true. That is, in order to test whether a hypothesis $\mathrm{HO}$ is correct, first assume that the hypothesis $\mathrm{HO}$ is correct, and then make a decision to accept or reject the hypothesis $\mathrm{H} 0$ according to the sample. If the observed value of the sample leads to the occurrence of a "small probability event," the hypothesis H0 should be rejected, otherwise the hypothesis $\mathrm{H} 0$ should be accepted. Because the correlation between the variables is not high, so multifactor analysis is not considered. Suppose H0 is the acceptance of the null hypothesis, $\mathrm{H} 1$ is the rejection of the null hypothesis, and the analysis results are as follows:

It can be seen from Table 2 that the significance of gender's one-way analysis of variance is 0.0001 , which is far less than 0.05 . It means that different genders are involved in entrepreneurial intentions.

It can be seen from Table 3 that men's entrepreneurial intentions are significantly higher than women's entrepreneurial intentions.

Figure 8 shows a smooth curve in the female correlation graph on the left, indicating that there is an exponential decline in the intention to IE. Linearity means that it is relatively stable, has a higher acceptance of IE education, and has more entrepreneurial ideas.

It can be seen from Table 4 that the significance $p$ of the one-way analysis of variance for the student origin is $0.1562>0.05$, which fails the significance test, that is, there is no significant difference in the entrepreneurial intentions of students from different student origins. 
TABLE 1: List of basic information.

\begin{tabular}{|c|c|c|c|}
\hline Basic situation & Options & Subtotal & Proportion (\%) \\
\hline \multirow{2}{*}{ Gender } & (A) Boy & 246 & 41 \\
\hline & (B) Girl & 354 & 59 \\
\hline \multirow{2}{*}{ Birthplace } & (A) Rural area & 207 & 34.40 \\
\hline & (B) City & 393 & 65.60 \\
\hline \multirow{2}{*}{ Is it a only child? } & (A) Yes & 380 & 63.40 \\
\hline & (B) No & 220 & 35.60 \\
\hline \multirow{4}{*}{ Grade } & (A) College freshman & 203 & 33.80 \\
\hline & (B) Sophomore & 375 & 62.50 \\
\hline & (C) Junior & 10 & 1.70 \\
\hline & (D) Senior year & 12 & 2.00 \\
\hline \multirow{2}{*}{ Have you received entrepreneurial education } & (A) Yes & 97 & 16.10 \\
\hline & (B) No & 503 & 83.90 \\
\hline \multirow{2}{*}{ Did you work part-time during university? } & (A) Yes & 183 & 30.40 \\
\hline & (B) No & 417 & 69.60 \\
\hline \multirow{2}{*}{ Entrepreneurship experience of parents and relatives } & (A) Yes & 224 & 37.20 \\
\hline & (B) No & 376 & 62.70 \\
\hline \multirow{4}{*}{ Father's education } & (A) University and above & 213 & 35.50 \\
\hline & (B) High school, technical secondary school & 217 & 36.10 \\
\hline & (C) Junior high school & 135 & 22.40 \\
\hline & (D) Elementary school and below & 36 & 6.00 \\
\hline \multirow{4}{*}{ Mother's education } & (A) University and above & 151 & 25.10 \\
\hline & (B) High school, technical secondary school & 235 & 39.10 \\
\hline & (C) Junior high school & 152 & 25.30 \\
\hline & (D) Elementary school and below & 64 & 10.50 \\
\hline \multirow{5}{*}{ Father's occupation } & (A) Institution or agency & 209 & 34.80 \\
\hline & (B) Contract enterprise unit & 128 & 21.20 \\
\hline & (C) Self-employed & 156 & 25.90 \\
\hline & (D) No job, laid-off job & 38 & 6.40 \\
\hline & (E) Farmer & 71 & 11.70 \\
\hline \multirow{5}{*}{ Mother's occupation } & (A) Institution or agency & 184 & 30.60 \\
\hline & (B) Contract enterprise unit & 112 & 18.60 \\
\hline & (C) Self-employed & 145 & 24.10 \\
\hline & (D) No job, laid-off job & 82 & 13.40 \\
\hline & (E) Farmer & 82 & 13.40 \\
\hline
\end{tabular}

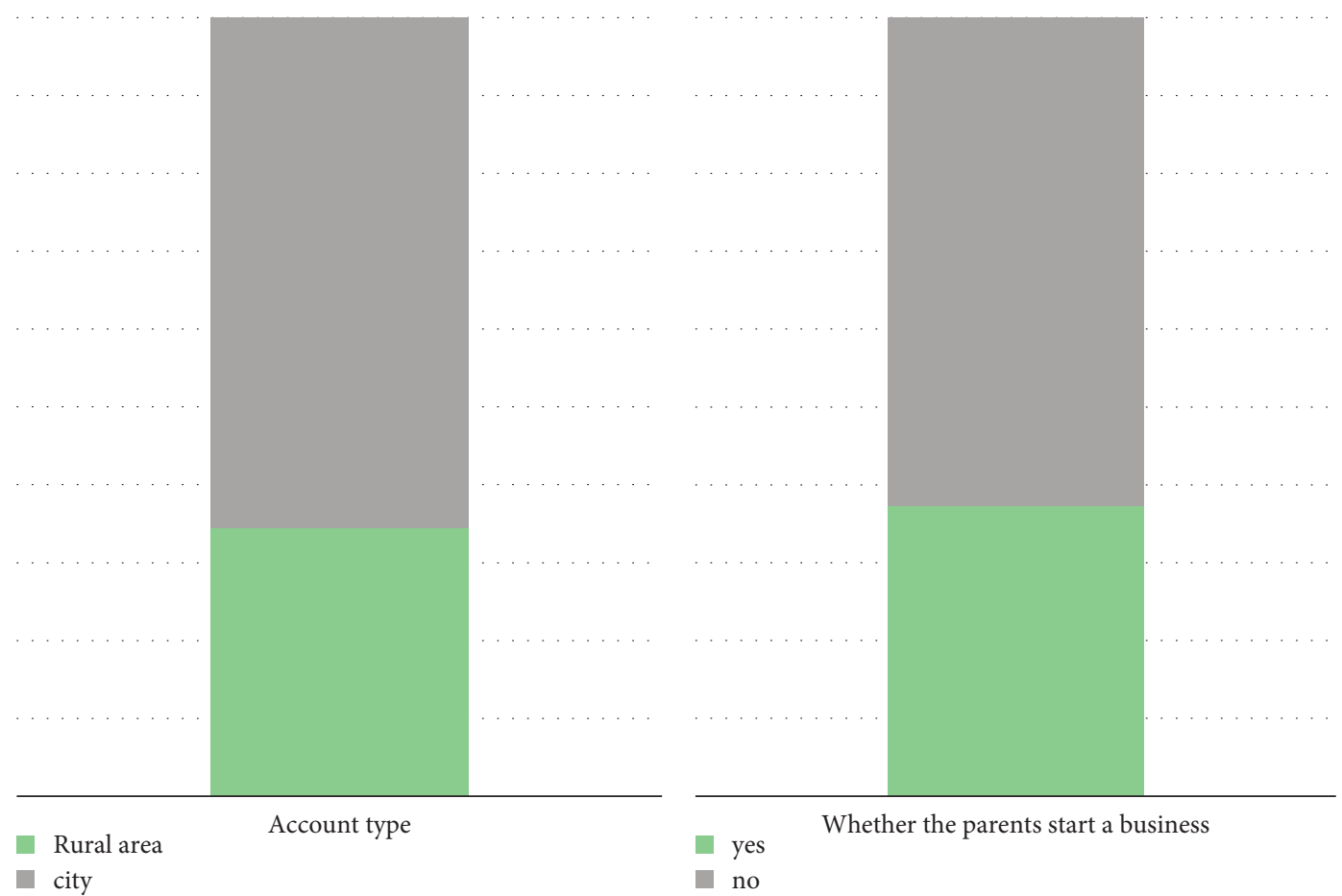

FIgURE 4: State distribution diagram. 


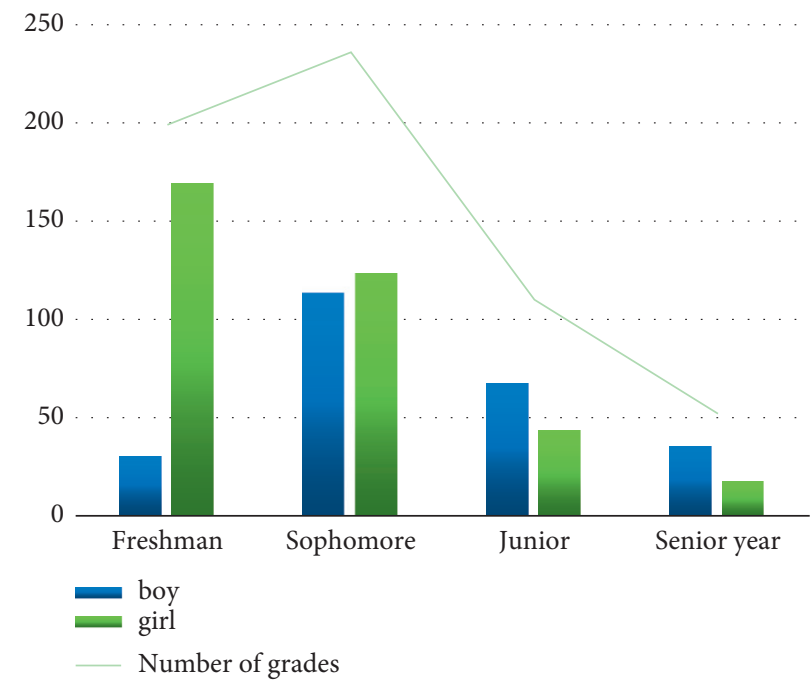

Figure 5: Gender distribution of the number of people.



Figure 6: Visualization of personal situation.

\subsubsection{One-Way Analysis of Variance of Parent Occupation.} It can be seen from Table 5 that, in the one-way analysis of variance of the parent's occupation, the significance test value of the father's occupation is $0.027<0.05$, which passes the significance test; and the significance test value of the mother's occupation is $0.136>0.05$, which means that the significance test is not passed. The difference of father's occupation has a significant influence on children's entrepreneurial intention, while the mother's occupation has no influence on children's entrepreneurial intention. From Table 6, it can be found that the children whose father is a self-employed entrepreneur have the highest entrepreneurial intention, which is much higher than the average level of the questionnaire, and the children whose father works in an agency or institution have the lowest entrepreneurial intention, which is lower than the evaluation level of the questionnaire survey results, as shown in Figure 9.
In the analysis of the correlation between parents' occupations and their children's innovation and entrepreneurial intentions, the residuals can be seen that the father's occupation has a greater impact on the children, while the mother's is relatively small.

3.2.4. Single-Factor Analysis of Entrepreneurial Experience of Parents and Relatives. It can be seen from Table 7 that the oneway ANOVA value of whether parents and relatives have entrepreneurial experience is 0.0032 , which is far less than 0.05 , thus passing the significance test; that is, whether the entrepreneurial experience of parents and relatives has a significant impact on the entrepreneurial intention of college students. It can be concluded that the average value of students whose parents and relatives have entrepreneurial experience is greater than that of students whose parents and relatives have no entrepreneurial experience, that is, college students whose parents and relatives have entrepreneurial experience have higher entrepreneurial intentions, as shown in Table 8.

It can be clearly found in Figure 10 that, when $r=0.8$ and $p=0.2$, as the father's entrepreneurial journey increases, the children's intention to innovate and start a business will gradually increase, but for the mother the impact is relatively small.

3.3. Research Results. For college students who want to start a business, the most important thing is to get rid of paper talk, act boldly, take advantage of the limited time and conditions of the university, and actively participate in related school club activities, such as entrepreneurship seminars, entrepreneur seminars, entrepreneurship competitions, entrepreneurship training camps, and entrepreneurship guidance courses, to strive for various opportunities to exercise and improve their entrepreneurial ability through participation. Students who participate in entrepreneurial activities or entrepreneurial competitions do not necessarily have very strong entrepreneurial intentions, but through such 

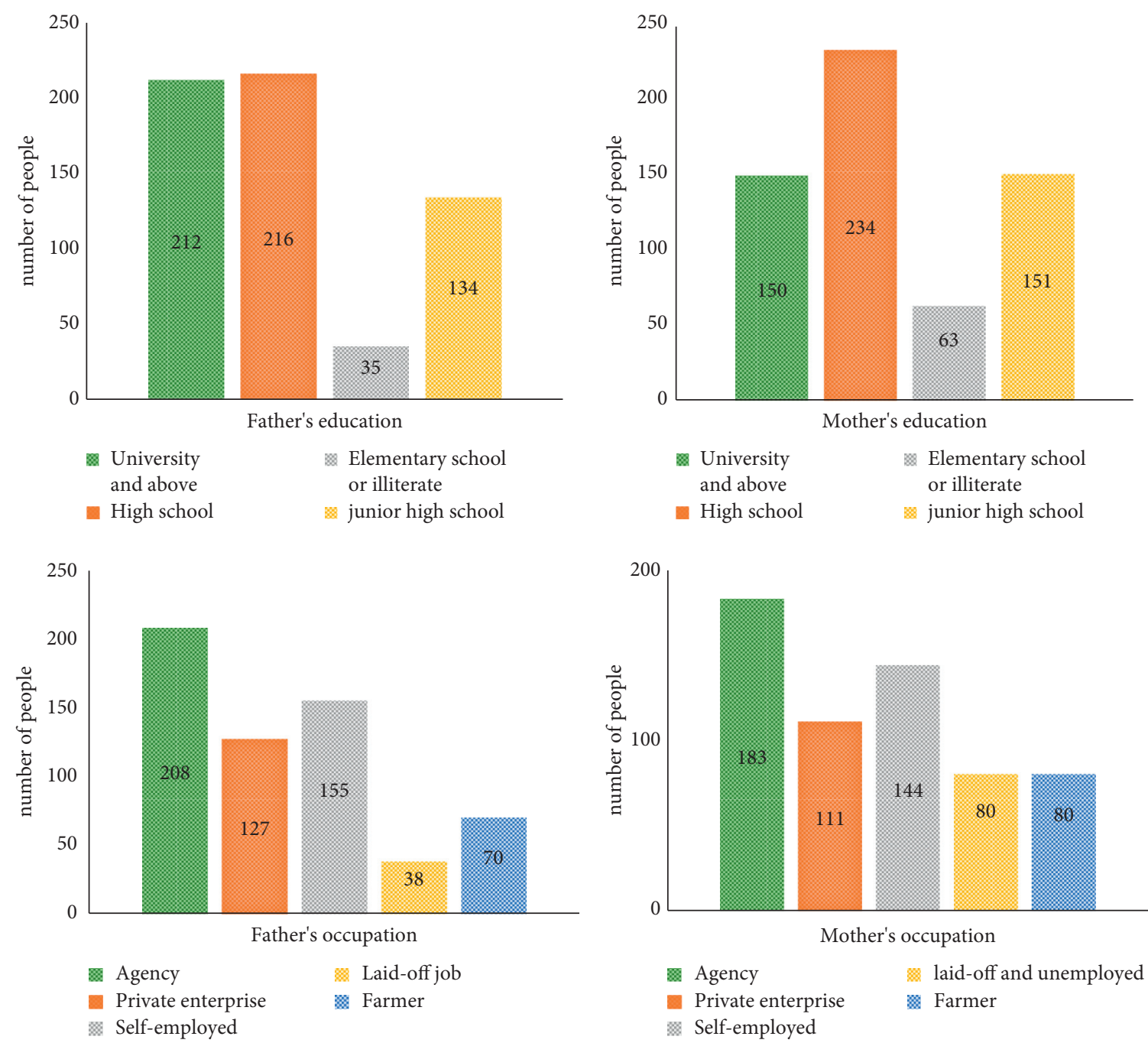

FIGURE 7: Summary of family situation.

TABLE 2: Gender one-way analysis of variance ANOVA.

\begin{tabular}{lcccc}
\hline & Sum of square & DF_value & Mean square & F_value \\
\hline B_groups & 311.349 & 1 & 311.349 & 10.51 \\
S_group & 17655.911 & 596 & 29.624 & 0.0001 \\
Sum & 17967.259 & 597 & & \\
\hline
\end{tabular}

TABle 3: Descriptive statistics of gender univariate analysis.

\begin{tabular}{lccccc}
\hline & $N$ & $M$ & S. deviation & S. error & \multicolumn{2}{c}{ 95\% confidence } \\
& & & & L_limit & 15.934 \\
Boy & 245 & 16.694 & 6.042 & 0.386 & 14.748 \\
Girl & 353 & 15.227 & 5.99 & 0.265 & 17.454 \\
Sum & 598 & 15.828 & 5.49 & 0.334 & 15.387 \\
\hline
\end{tabular}

entrepreneurial activities they can accumulate certain entrepreneurial knowledge and entrepreneurial resources, which will increase the possibility of entrepreneurship. Participating in entrepreneurial activities provides students with a good opportunity to contact society and entrepreneurship, which can help students open the door to entrepreneurship. You can also step into society, do market research, understand social needs, apply what you have learned to real life, integrate theory with practice, practice in learning, and learn in practice. Through experiments, it is clear that the impact of IE education is specifically the following aspects. Educational methods account for $63.8 \%$; parental environment accounts for $13.4 \%$, and personal effort for $22.4 \%$. 


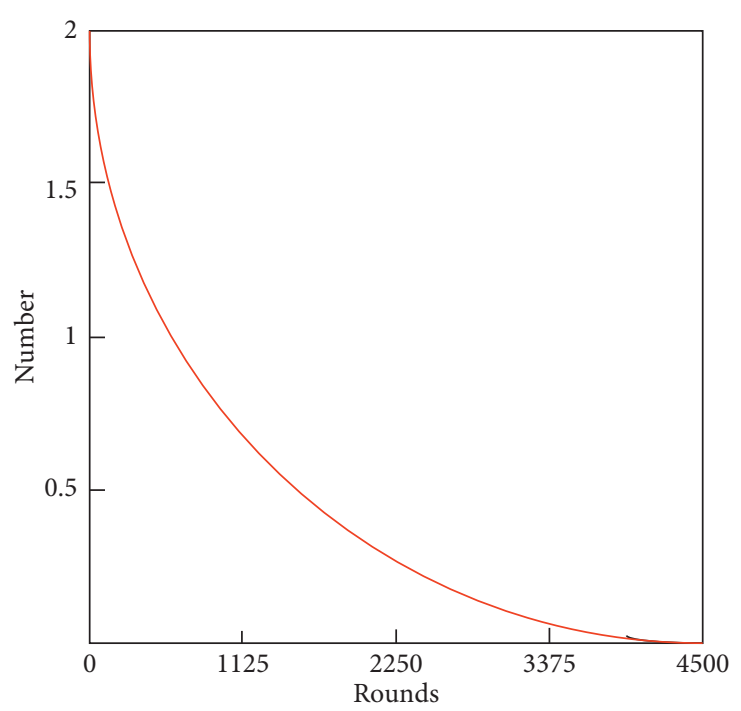

GIRL

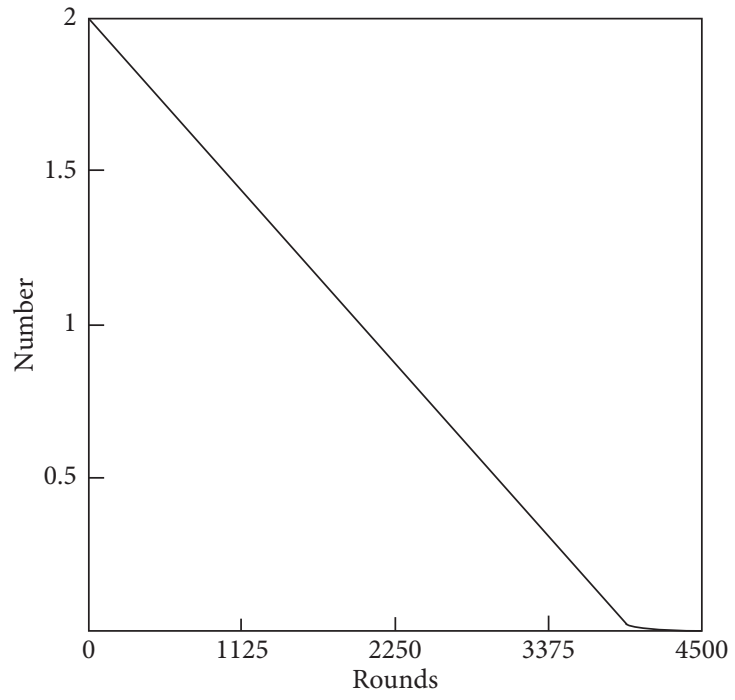

BOY

Figure 8: Gender-related residual graph.

TABLE 4: One-way analysis of variance (ANOVA).

\begin{tabular}{|c|c|c|c|c|c|}
\hline & Sum of square & DF_value & Mean square & $F \_$value & Significance \\
\hline B_groups & 60.627 & 1 & 60.627 & 2.018 & 0.1562 \\
\hline S_group & 17906.632 & 596 & 30.045 & & \\
\hline Sum & 17967.259 & 597 & & & \\
\hline
\end{tabular}

TABLE 5: One-way analysis of variance (ANOVA) of parent occupation.

\begin{tabular}{|c|c|c|c|c|c|}
\hline Father's occupation & Sum of square & DF_value & Mean square & $F \_$value & Significance \\
\hline B_groups & 328.494 & 4 & 82.124 & 2.761 & 0.027 \\
\hline S_group & 17638.765 & 593 & 29.745 & & \\
\hline Sum & 17967.259 & 597 & & & \\
\hline Mother occupation & Sum of square & DF_value & Mean square & $F \_$value & Significance \\
\hline B_groups & 210.609 & 4 & 52.652 & 1.758 & 0.136 \\
\hline S_group & 17756.65 & 593 & 29.944 & & \\
\hline Sum & 17967.259 & 597 & & & \\
\hline
\end{tabular}

TABLE 6: Descriptive statistics of single factor analysis of father's occupation.

\begin{tabular}{|c|c|c|c|c|c|c|}
\hline \multirow{2}{*}{ Father's occupation } & \multirow{2}{*}{$N$} & \multirow{2}{*}{$M$} & \multirow{2}{*}{ S. deviation } & \multirow{2}{*}{ S. error } & \multicolumn{2}{|c|}{ 95\% confidence } \\
\hline & & & & & L_limit & U_limit \\
\hline Institution or agency & 208 & 15.019 & 5.62 & 0.39 & 14.251 & 15.787 \\
\hline Contract enterprise unit & 127 & 16.063 & 5.217 & 0.463 & 15.147 & 1.979 \\
\hline Self-employed & 155 & 16.852 & 5.146 & 0.413 & 16.035 & 17.668 \\
\hline No job, laid-off job & 38 & 16.211 & 6.968 & 1.13 & 13.92 & 18.501 \\
\hline Farmer & 70 & 15.329 & 5.1039 & 0.61 & 14.112 & 16.546 \\
\hline Sum & 598 & 15.828 & 5.486 & 0.2243 & 15.387 & 16.268 \\
\hline
\end{tabular}

\section{Discussion}

Contemporary college students are even taking advantage of the spring breeze of reform, and they must also actively master innovative and entrepreneurial skills, apply their knowledge to the real, exercise their courage, and strive to be pioneers of the times.
This study examines the relationship between entrepreneurial education and opportunistic entrepreneurial intention from the perspective of empirical analysis. However, due to the limitations of personal ability and objective environment, this study has the following limitations. (1) Sample factors limitation: in this study, only undergraduates from Huazhong Agricultural University 

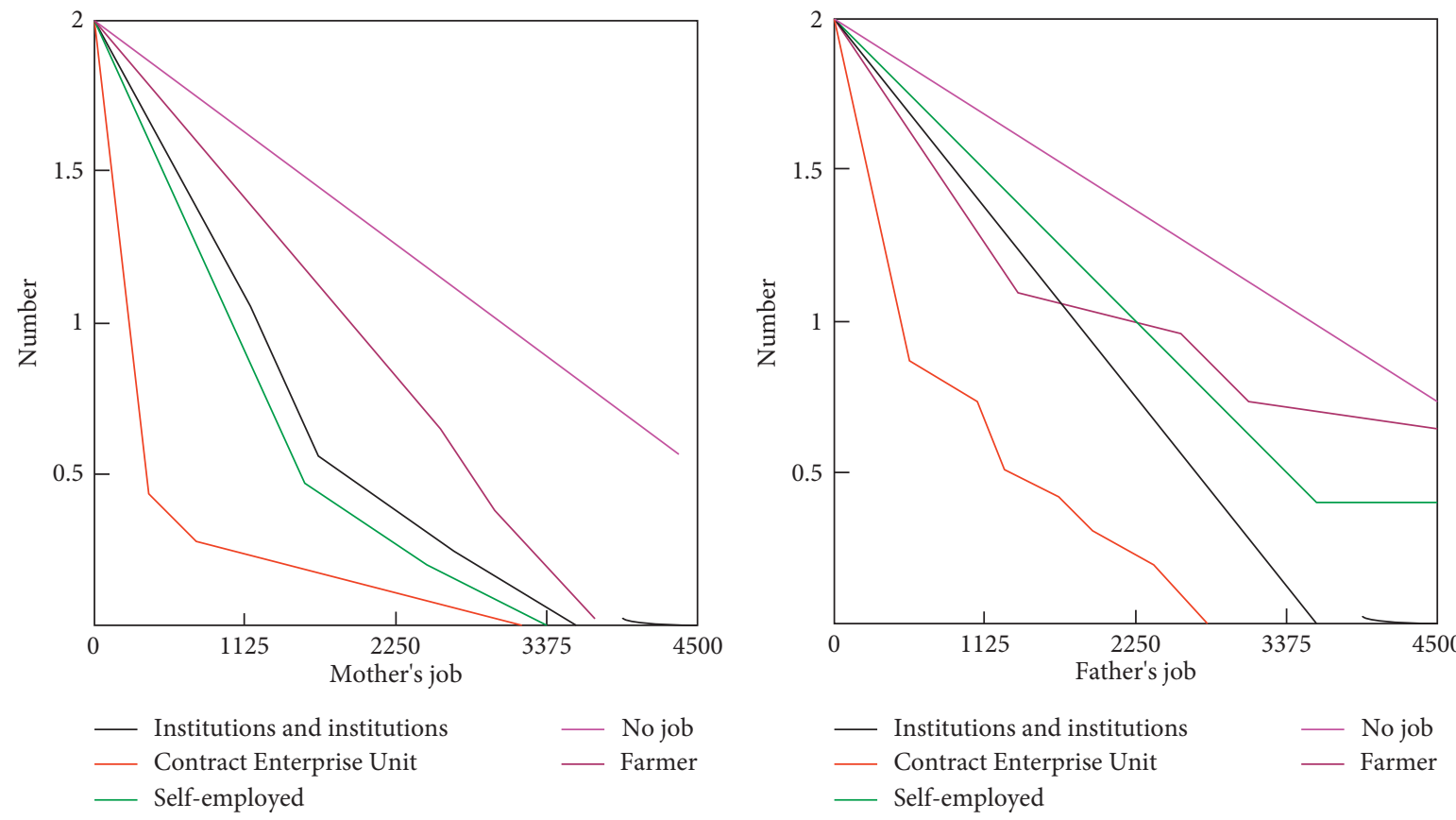

FIGURE 9: Occupation-related residual diagram.

TABLE 7: One-way analysis of variance (ANOVA) of parents' and relatives' entrepreneurial experience.

\begin{tabular}{|c|c|c|c|c|c|}
\hline & Sum of square & $d f$ & Mean square & $F$ & Significance \\
\hline Between groups & 343.729 & 2 & 171.865 & 5.802 & 0.0032 \\
\hline Squares within group & 17623.53 & 595 & 29.619 & & \\
\hline Sum & 17967.259 & 597 & & & \\
\hline
\end{tabular}

TABLE 8: Descriptive statistics of single-factor analysis of entrepreneurial experience of parents and relatives.

\begin{tabular}{lccccc}
\hline Entrepreneurship experience & \multirow{2}{*}{$N$} & $M$ & S. deviation & S. error & \multicolumn{2}{c}{$\begin{array}{c}\text { 95\% confidence } \\
\text { L_limit }\end{array}$} \\
\hline Yes & & & 5.631 & 0.371 & 15.949 \\
No & 222 & 16.68 & 5.389 & 0.279 & 14.802 \\
Sum & 375 & 15.349 & 5.486 & 0.2243 & 15387 \\
\hline
\end{tabular}

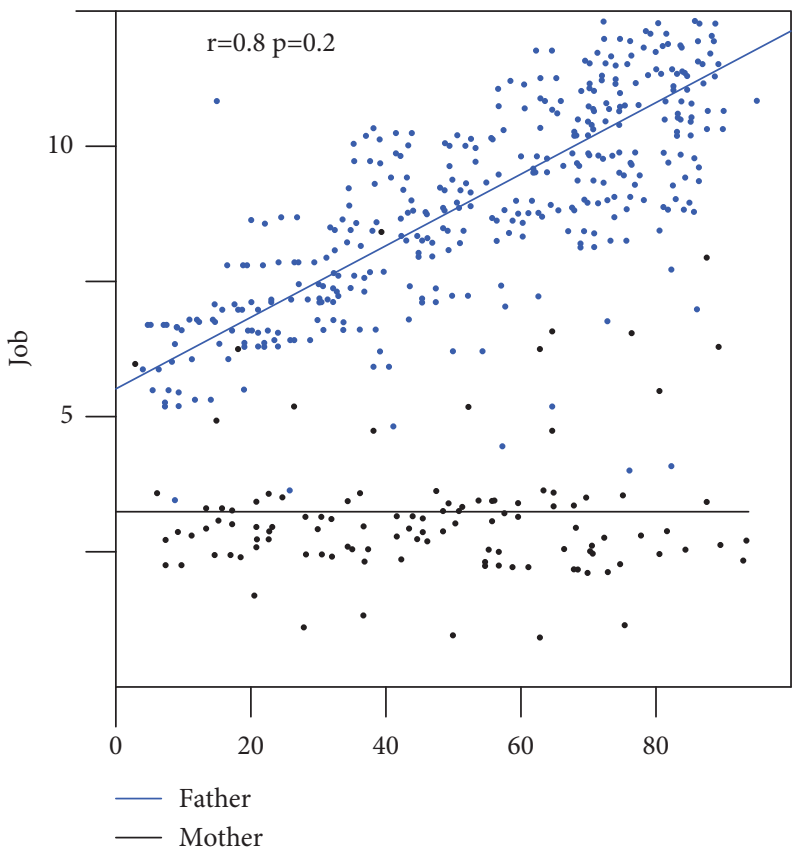

FIgURE 10: Parent entrepreneurship influence diagram. 
were selected as the survey subjects. The geographical factors were not overcome, and the questionnaires were distributed nationwide, and there is no questionnaire survey for graduate students, which made the sample size insufficient to represent the whole country. (2) Lack of in-depth research: due to space limitations, this article is more about investigating college students' own abilities and experience, and the entrepreneurial experience of their parents, that is, internal influence factors. It does not focus on the feedback from the big data platform. (3) The research methods have certain limitations. This article mainly adopts the questionnaire survey method, which is more targeted at college students. It has limitations in sample selection and involves less theoretical research. It only reviews the theory in the literature review, which, to a certain extent, contributes to the comprehensiveness of this article. And accuracy has an impact.

Hope that these problems can be solved in the follow-up study and research.

\section{Conclusions}

The current trend of big data platform infrastructure construction is cloudification and openness. This platform needs to be able to provide all kinds of big data-related PaaS services, and it also needs to enable simple and flexible combinations of various services to meet changing and customized needs. How to provide a flexible, agile, yet stable and high-performance big data platform on the cloud? How to efficiently use the characteristics of cloud computing to develop a big data platform? It is worth thinking about. Entrepreneurship education was developed in the United States in the last century, and the effect was remarkable, so it developed rapidly in many countries. At the same time, the upgrade of cloud computing and big data platforms is also relatively hot. Since the end of the last century, more and more topics have been focused on college students' IE education, and they have immediately become a hot issue of domestic public and social concern, as well as the focus of my country's higher education theory and practice. This has become a sharp sword for how to solve the employment problem and use the vigor, courage, and knowledge of college students to vigorously develop IE education. At the same time, this is also a matter of everyone and the society. Therefore, IE education also requires our cooperation. Among them, college education is the only way for most people to receive IE education. Therefore, the significance of this topic is very important in the context of the times. With the platform construction, IE education will be more standardized and simple.

\section{Data Availability}

No data were used to support this study.

\section{Conflicts of Interest}

The authors declare that there are no conflicts of interest.

\section{Authors' Contributions}

All the authors have read and approved the manuscript.

\section{Acknowledgments}

This work was supported by the Outstanding Youth Project of Scientific Research Project of Hunan Education Department "Study on the adaptability of university entrepreneurship tutors under the background of Moral education" (20B339).

\section{References}

[1] M. Peris-Ortiz, J. A. Gómez, and J. M. Merigó-Lindahl, "Entrepreneurial universities," Innovation, Technology, and Knowledge Management, pp. 287-302, 2017.

[2] W. Wei, X. Fan, and H. Song, "Imperfect information dynamic Stackelberg game based resource allocation using hidden Markov for cloud computing," IE educationE Transactions on Services Computing, vol. 11, no. 99, pp. 78-89, 2018.

[3] W. Zhang, Y. Lin, S. Xiao, J. Wu, and S. Zhou, "Privacy preserving ranked multi-keyword search for multiple data owners in cloud computing," IEEE Transactions on Computers, vol. 65, no. 5, pp. 1566-1577, 2016.

[4] L. Jin, Y. Zhang, and X. Chen, "Secure attribute-based data sharing for resource-limited users in cloud computing," Computers \& Security, vol. 72, no. JAN, pp. 1-12, 2018.

[5] S. Li and J. Feng, "An optimized data processing model for computer big data platform based on parallel computing," Boletin Tecnico/technical Bulletin, vol. 55, no. 8, pp. 318-324, 2017.

[6] S. Martin, B. Peter, and S. Miroslav, "Big data processing and analytics platform architecture for process industry factories," Big Data \& Cognitive Computing, vol. 2, no. 1, p. 3, 2018.

[7] E. B. Sifah, H. Xia, and C. Cobblah, "BEMPAS: a decentralized employee performance assessment system based on blockchain for smart city governance," IE educationE Access, vol. 99, p. 1, 2020.

[8] H. A. Li, M. Zhang, and K. Yu, "R3MR: region growing based 3D mesh reconstruction for big data platform," IE educationE Access, vol. 99, p. 1, 2020.

[9] P. Shan, "Image segmentation method based on K-mean algorithm," EURASIP Journal on Image and Video Processing, vol. 2018, no. 1, p. 81, 2018.

[10] F. Zhao, L. Y. Zhang, and M. M. Zhao, "Architecture and technical exploration of big data platform for ecological environment," Chinese Journal of Ecology, vol. 36, no. 3, pp. 824-832, 2017.

[11] I. Polychronou, G. Stoitsis, M. Papakonstantinou, and N. Manouselis, "Stress-testing big data platform to extract smart and interoperable food safety analytics," International Journal of Metadata Semantics and Ontologies, vol. 14, no. 4, p. 306, 2020.

[12] Y. X. Sun, P. Shen, and J. Y. Zhang, "Epidemiological characteristics of COVID-19 monitoring cases in Yinzhou district based on health big data platform," Zhonghua liu xing bing xue za zhi= Zhonghua liuxingbingxue zazhi, vol. 41, no. 8, pp. 1220-1224, 2020.

[13] M. Joolfoo, R. A. Jugurnauth, and K. Joolfoo, "Customer churn prediction in telecom using machine learning in big data platform," Journal of Critical Reviews, vol. 7, no. 11, p. $1991,2020$.

[14] C. Esposito, M. Ficco, and B. B. Gupta, "Blockchain-based authentication and authorization for smart city applications," Information Processing \& Management, vol. 58, no. 2, p. 102468, 2021. 
[15] Y. L. Chen, H. Wang, and Y. N. Zhou, "Epidemiological characteristics of COVID-19 in wuchang district of wuhan," Zhonghua liu xing bing xue za zhi= Zhonghua liuxingbingxue zazhi, vol. 41, no. 10, pp. 1616-1622, 2020.

[16] H. Mandong, Y. Zhong, X. Shuxuan, L. Haibin, and L. Zhihan, "Fuzzy system based medical image processing for brain disease prediction," Frontiers in Neuroscience, vol. 2021, p. 965, 2021.

[17] R. Deng, R. Lu, and C. Lai, "Optimal workload allocation in fog-cloud computing toward balanced delay and power consumption," IE educationE Internet of Things Journal, vol. 3, no. 6, pp. 1171-1181, 2017.

[18] F. Jalali, K. Hinton, R. Ayre, T. Alpcan, and R. S. Tucker, "Fog computing may help to save energy in cloud computing," IEEE Journal on Selected Areas in Communications, vol. 34, no. 5, pp. 1728-1739, 2016.

[19] J. L. Tsai and N. W. Lo, "A privacy-aware authentication scheme for distributed mobile cloud computing services," IE educationE Systems Journal, vol. 9, no. 3, pp. 805-815, 2017.

[20] Y. Al-Dhuraibi, F. Paraiso, and N. Djarallah, "Elasticity in cloud computing: state of the art and research challenges," $I E$ educationE Transactions on Services Computing, vol. 99, p. 1, 2017.

[21] W. Tang and W. Feng, "Parallel map projection of vectorbased big spatial data: coupling cloud computing with graphics processing units," Computers, Environment and Urban Systems, vol. 61, pp. 187-197, 2017.

[22] P. S. Pillai and S. Rao, "Resource allocation in cloud computing using the uncertainty principle of game theory," IEEE Systems Journal, vol. 10, no. 2, pp. 637-648, 2016. 